\title{
The structure of lean hydrogen-air flame balls
}

\author{
E. Ferna'ndez-Tarrazo, A.L. Sanchez, A. Liñan, F.A. Williams
}

\begin{abstract}
An analysis of the structure of flame balls encountered under microgravity conditions, which are stable due to radiant energy losses from $\mathrm{H}_{2} \mathrm{O}$, is carried out for fuel-lean hydrogen-air mixtures. It is seen that, because of radiation losses, in stable flame balls the maximum flame temperature remains close to the crossover temperature, at which the rate of the branching step $\mathrm{H}+\mathrm{O}_{2} \rightarrow \mathrm{OH}+\mathrm{O}$ equals that of the recombination step $\mathrm{H}+\mathrm{O}_{2}+\mathrm{M} \rightarrow \mathrm{HO}_{2}+\mathrm{M}$. Under those conditions, all chemical intermediates have very small concentrations and follow the steady-state approximation, while the main species react according to the overall step $2 \mathrm{H}_{2}+\mathrm{O}_{2} \rightarrow 2 \mathrm{H}_{2} \mathrm{O}$; so that a one-step chemical-kinetic description, recently derived by asymptotic analysis for near-limit fuel-lean deflagrations, can be used with excellent accuracy to describe the whole branch of stable flame balls. Besides molecular diffusion in a binary-diffusion approximation, Soret diffusion is included, since this exerts a nonnegligible effect to extend the flammability range. When the large value of the activation energy of the overall reaction is taken into account, the leading-order anal-ysis in the reaction-sheet approximation is seen to determine the flame ball radius as that required for radi-ant heat losses to remove enough of the heat released by chemical reaction at the flame to keep the flame temperature at a value close to crossover. The results are relevant to burning velocities at lean equivalent ratios and may influence fire-safety issues associated with hydrogen utilization.
\end{abstract}

\section{Introduction}

The increased interest in hydrogen usage focuses attention on safety concerns such as the possibility of sustained fires developing in systems having very low concentrations of hydrogen in air. Hydrogen deflagrations persist to much lower concentrations than would be possible under steady, planar conditions [1] because of hydrogen's strong propensity for diffusive-thermal instability. Flame balls constitute limiting configurations in which hydrogen combustion, dominated in this case by its high diffusivity leading to higher flame temperatures, can occur under the leanest possible conditions [2]. Collections of flame balls can continue to burn for long periods of time in mixtures with insufficient hydrogen for ordinary flame propagation [3]. For these reasons, in considering fire hazards, it is desirable to have accurate descriptions 
of flame-ball structures in very lean hydrogen-air mixtures. The study reported here was performed in an effort to improve such descriptions and to be better able to understand and to calculate the resulting flammability behavior.

Computational methods are sufficiently advanced that flame-ball structures can be addressed numerically $[4,5]$. While numerical approaches can include many phenomena, they often are not well suited to specifically identifying the most important ones. Asymptotic and analytic methods, on the other hand, can isolate the most important effects, thereby increasing understanding of the flame structures significantly. In addition, they often can yield formulas that are readily applied to calculate quantities of interest in applications. For these reasons, in the present work a simplified analysis is derived for better understanding of near-flammability combustion and flame-ball burning.

\section{Formulation}

\subsection{Starting equations}

The problem to be addressed is flame-ball combustion of lean hydrogen-air mixtures in a gravity-free environment, which can be steady and spherically symmetrical. Then the radial velocity of the fluid is zero, so that momentum conservation implies that the pressure $p$ is constant. The radial variation of the temperature and composition is obtained by integrating the conservation equations for energy and chemical species

$\frac{1}{r^{2}} \frac{\mathrm{d}}{\mathrm{d} r}\left[\lambda r^{2} \frac{\mathrm{d} T}{\mathrm{~d} r}\right]=Q_{R}-\sum_{i} h_{i}^{o} \dot{m}_{i}$

$\frac{1}{r^{2}} \frac{\mathrm{d}}{\mathrm{d} r}\left[\rho D_{i} r^{2}\left(\frac{\mathrm{d} Y_{i}}{\mathrm{~d} r}+\frac{\alpha_{i} Y_{i}}{T} \frac{\mathrm{d} T}{\mathrm{~d} r}\right)\right]=\dot{m}_{i}$,

with boundary conditions

$\frac{\mathrm{d} T}{\mathrm{~d} r}=\frac{\mathrm{d} Y_{i}}{\mathrm{~d} r}=0 \quad$ at $r=0$

and

$T-T_{\infty}=Y_{i}-Y_{i \infty}=0 \quad$ as $r \rightarrow \infty$,

where the subscript $\infty$ denotes ambient quantities, $r$ is the distance to the flame-ball center, and $T, \rho$ and $\lambda$ are the temperature, density and thermal conductivity of the gas mixture. The composition is determined in terms of the mass fraction $Y_{i}$ of each species $i$, whose molecular diffusivity and enthalpy of formation are denoted by $D_{i}$ and $h_{i}^{\circ}$, respectively.

The computation of the mass rate of production of species $i$, denoted by $\dot{m}_{i}$, requires specification of the chemical-reaction scheme, an appropriate choice being the so-called San Diego Mechanism [6] of 21 steps, whose accuracy in describing hydro- gen-air combustion has been tested thoroughly [1]. In fuel-lean hydrogen-air flame balls, the thermal conductivity is dominated by the values of nitrogen and oxygen, so that the approximate expression

$\lambda / c_{p}=2.58 \times 10^{-5}(T / 298)^{0.7} \mathrm{~kg} /(\mathrm{ms})$,

suggested in [7] for methane-air flames, can be used with excellent accuracy to compute $\lambda$, with the value of the specific heat at constant pressure $c_{p}=\sum_{i} c_{p i} Y_{i}$ evaluated with use made of NASA polynomial fits for the temperature dependence of each $c_{p i}$. At the same level of approximation [7], $D_{i}$ can be computed as the binary diffusion coefficient of the species $i$ into nitrogen according to

$\rho D_{i}=2.58 \times 10^{-5}(T / 298)^{0.7} / L_{i}, \mathrm{~kg} /(\mathrm{ms})$

with $L_{i}=(1.11,0.30,0.83)$ for $\left(\mathrm{O}_{2}, \mathrm{H}_{2}, \mathrm{H}_{2} \mathrm{O}\right)$, respectively. Thermal diffusion is negligible for all species, except for $\mathrm{H}$ and $\mathrm{H}_{2}$, for which the Soret factors are given by $\alpha_{\mathrm{H}}=-0.23$ and $\alpha_{\mathrm{H}_{2}}=-0.29$ [8]. A detailed description is also needed for the rate of radiant energy loss per unit volume $Q_{R}$, which is the divergence of the radiant-energy flux vector. For instance, if a differential gray-gas approximation [9] is adopted, $Q_{R}$ is a function of the mean intensity of radiation $I=I_{\mathrm{A}} /(4 \pi)$ that can be expressed in the form $Q_{R}=-\alpha_{i}\left(I_{\mathrm{A}}-4 \sigma T^{4}\right)$, where $I_{\mathrm{A}}$ satisfies the equation

$\frac{1}{r^{2}} \frac{\mathrm{d}}{\mathrm{d} r}\left[\frac{r^{2}}{3 \alpha_{a}} \frac{\mathrm{d} I_{\mathrm{A}}}{\mathrm{d} r}\right]=\alpha_{a}\left(I_{\mathrm{A}}-4 \sigma T^{4}\right)$

with boundary conditions $\mathrm{d} I_{\mathrm{A}} / \mathrm{d} r=0$ at $r=0$ and $I_{\mathrm{A}}-4 \sigma T_{\infty}^{4}=0$ as $r \rightarrow \infty$. Here, $\sigma$ denotes the Stefan-Boltzmann constant and $\alpha_{a}=\kappa_{\mathrm{H}_{2} \mathrm{O}} p$ $\left(W / W_{\mathrm{H}_{2} \mathrm{O}}\right) Y_{\mathrm{H}_{2} \mathrm{O}}$ is the gray mean value of the absorption coefficient corresponding to the main contributing species $\mathrm{H}_{2} \mathrm{O}$, where $W=$ $\left(\sum_{i} Y_{i} / W_{i}\right)^{-1}$ is the mean molecular weight, computed from the specific molecular weights $W_{i}$ of the different chemical species $i$. The Plank-mean absorption coefficient $\kappa_{\mathrm{H}_{2} \mathrm{O}}$ is a function of the temperature, given for instance in [10], that in the range of temperatures of interest $(270<$ $T<1300 \mathrm{~K}$ ) can be shown to be accurately represented by the expression $\kappa_{\mathrm{H}_{2} O}=5.72 \times 10^{-4}$ $(T / 298)^{-2} \mathrm{~s}^{2} / \mathrm{kg}$.

The inverse of $\alpha_{a}$ gives the characteristic value of the absorption length, which can be estimated from the above expressions to be of the order $\alpha_{a}^{-1} \simeq 100 \mathrm{~cm}$ for characteristic flame-ball combustion conditions (e.g., $\alpha_{a}^{-1}=92 \mathrm{~cm}$ for $T=$ $\left.1000 \mathrm{~K}, p=1 \mathrm{~atm}, Y_{\mathrm{H}_{2} \mathrm{O}}=0.125\right)$, a quantity that is therefore very large compared with the characteristic flame-ball radius $r_{f} \sim 1 \mathrm{~cm}$. According to (7), in the optically thin limit of small Bouguer numbers $\alpha_{a} r_{f} \ll 1$ that applies to flame-ball radiation, the variations of $I_{\mathrm{A}}$ from its boundary value $4 \sigma T_{\infty}^{4}$ are of order $\left(\alpha_{a} r_{f}\right)^{2} 4 \sigma T^{4}$ and can be therefore neglected in the first approximation when 
computing the radiant energy loss per unit volume; this leads to the familiar approximation

$Q_{R}=4 \kappa_{\mathrm{H}_{2} \mathrm{O}} \sigma p\left(W / W_{\mathrm{H}_{2} \mathrm{O}}\right) Y_{\mathrm{H}_{2} \mathrm{O}}\left(T^{4}-T_{\infty}^{4}\right)$,

to be employed in the analysis below.

\subsection{Sample numerical results}

The above equations were used to compute hydrogen-air flame balls at atmospheric pressure with $T_{\infty}=300 \mathrm{~K}$, giving the results shown in Figs. 1-3. For given ambient conditions, the flame-ball problem is known to possess two different solutions for values of the equivalence ratio within the flammability range. The plots show results corresponding to the stable branch of large flame balls, which begins at the flame-ball lean limit $\phi_{l}=0.0735$. Note that, because of the large fuel diffusivity, this critical value $\phi_{l}=0.0735$ is much smaller than that of planar flames for the same ambient conditions $\phi_{l}=0.251[13,14]$.

The solid curves in the plots of Figs. 1 and 2 represent the variation with equivalence ratio $\phi=8 Y_{\mathrm{H}_{2 \infty}} / Y_{\mathrm{O}_{2 \infty}}$ of the flame-ball radius $r_{f}$ and of the peak temperature $T_{\max }$, with the former plot including also results of experiments $[11,12]$. To be consistent with the reduced-chemistry results presented below, the value of $r_{f}$ is defined in the numerical computations as the radial location where the temperature takes its crossover value (given approximately by $T_{c}=1000 \mathrm{~K}$ at atmospheric pressure, as obtained from Eq. (10)), although the values determined with a different criterion (e.g., location of peak $\mathrm{H}_{2}$-consumption rate or maximum $\mathrm{H}$-atom concentration) would be very similar, especially for the larger values of $r_{f}$ corresponding to the stable branch away from the lean flammability limit. Sample profiles of main species and temperature are shown in Fig. 3 for a flame ball with $\phi=0.15$. To exhibit the existence of a concentrated reaction layer, to be used in deriving the analytical results, the plot includes also the local fuel burning rate (mass per unit volume per unit time) as evaluated from the rates of the different elementary reactions that involve the $\mathrm{H}_{2}$ molecule.

\subsection{One-step chemistry description}

As seen in Fig. 2, the solutions of stable flame balls corresponding to points along the upper branch of the $r_{f}-\phi$ curve exhibit moderate peak temperatures not far from the crossover value. As shown recently $[13,14]$, under those conditions all intermediates appear in very small concentrations and can be assumed to follow a steady-state description, whereas the main species react according to the overall reaction $2 \mathrm{H}_{2}+\mathrm{O}_{2} \rightarrow$ $2 \mathrm{H}_{2} \mathrm{O}$, with a rate (moles per unit volume per unit time) given by

$\omega=\frac{1}{G H}\left(\frac{k_{1 f}}{\alpha k_{4 f} C_{\mathrm{M}}}-1\right) \frac{k_{2 f} k_{3 f}}{k_{1 b}}\left(\rho Y_{\mathrm{H}_{2}} / W_{\mathrm{H}_{2}}\right)^{2}$,

as obtained from the seven elementary reactions $\mathrm{H}+\mathrm{O}_{2} \rightleftharpoons \mathrm{OH}+\mathrm{O}, \quad \mathrm{H}_{2}+\mathrm{O} \rightleftharpoons \mathrm{OH}+\mathrm{H}, \quad \mathrm{H}_{2}+$ $\mathrm{OH} \stackrel{2}{\rightleftharpoons} \mathrm{H}_{2} \mathrm{O}+\mathrm{H}, \quad \mathrm{H}+\mathrm{O}_{2}+\mathrm{M} \stackrel{4 f}{\rightarrow}{ }_{6 f} \mathrm{HO}_{2}+\mathrm{M}$, $\begin{array}{lll}\mathrm{HO}_{2}+\mathrm{H} \stackrel{5 f}{\rightarrow} \mathrm{OH}+\mathrm{OH}, \quad \mathrm{HO}_{2}+\mathrm{H} \stackrel{6 f}{\rightarrow} & \mathrm{H}_{2}+\mathrm{O}_{2},\end{array}$ and $\mathrm{HO}_{2}+\mathrm{OH} \stackrel{7 f}{\rightarrow} \mathrm{H}_{2} \mathrm{O}+\mathrm{O}_{2}$ by introducing steady state assumptions for the intermediaries $\mathrm{H}, \mathrm{O}$, $\mathrm{OH}$ and $\mathrm{HO}_{2}$. As noted in [13], the rate (9) applies with excellent accuracy at atmospheric and moder-

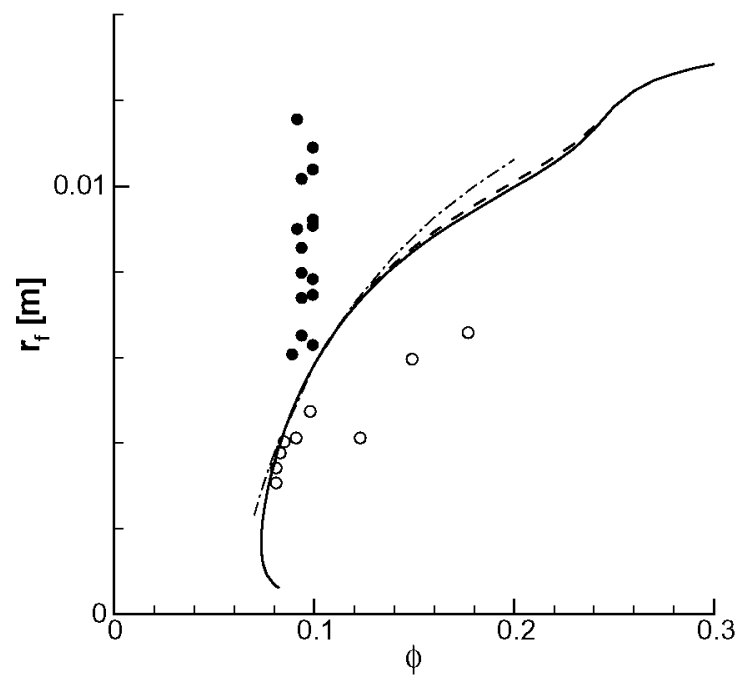

Fig. 1. The variation with equivalence ratio of the hydrogen-air flame-ball radius $r_{f}$ for $p=1$ atm and $T_{\infty}=300 \mathrm{~K}$ as obtained from experiments (hollow symbols: [11]; solid symbols: [12]), from computations with 21-step chemistry (solid curve) and with one-step chemistry (dashed curve) and from the reaction-sheet analysis (dot-dahed curve). 


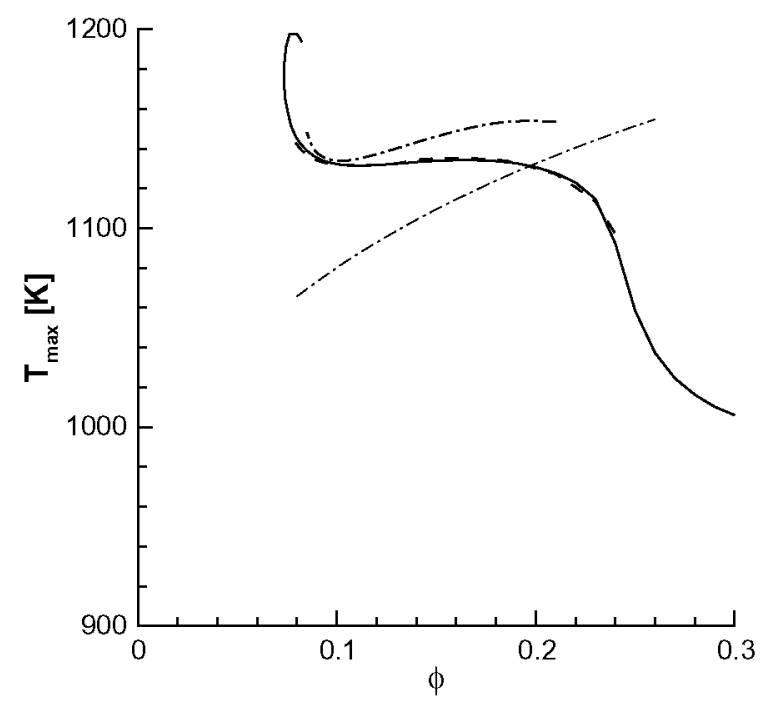

Fig. 2. The variation with equivalence ratio of the peak temperature $T_{\max }$ for the hydrogen-air flame ball with $p=1 \mathrm{~atm}$ and $T_{\infty}=300 \mathrm{~K}$ as obtained from computations with 21-step chemistry (solid curve) and with one-step chemistry (dashed curve) along with the results of the reaction-sheet analysis (dot-dashed curves), including the leading-order prediction $T_{\max }=T_{c}^{0}$ (thin curve) and the corrected value (thick curve).

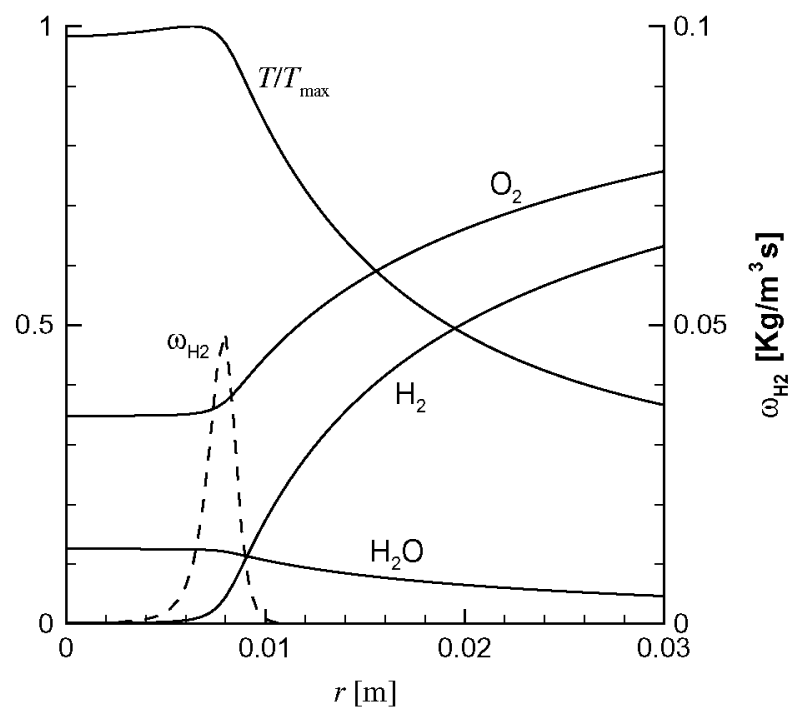

Fig. 3. Radial distributions of $T / T_{\max }, Y_{\mathrm{H}_{2}} / Y_{\mathrm{H}_{2 \infty}}, Y_{\mathrm{O}_{2}} / Y_{\mathrm{O}_{2 \infty}}$ and $Y_{\mathrm{H}_{2} \mathrm{O}}$ as obtained from numerical integrations with 21step chemistry for $\phi=0.15, T_{\infty}=300 \mathrm{~K}$ and $p=1$ (solid curves); the dashed line shows the radial variation of the fuel mass consumption rate.

ately elevated pressures, but for larger pressures approaching the third explosion limit the description should be modified to account for $\mathrm{H}_{2} \mathrm{O}_{2}$ formation from $\mathrm{HO}_{2}$ and its regeneration of active radicals. In the formulation, $C_{\mathrm{M}}$ represents the effective third-body concentration, which accounts for the non-unity third-body chaperon efficiencies of $\mathrm{H}_{2} \mathrm{O}$ and $\mathrm{H}_{2}$ associated with reaction $4 f$. The subscripts $f$ and $b$ in (9) denote forward and backward reaction-rate constants, while $G, H$ and $\alpha$ are functions of order unity of the composition and temperature [13].

The reaction rate (9) applies for temperatures larger than a crossover value $T_{c}$ for which

$k_{1 f}=\alpha k_{4 f} C_{\mathrm{M}}$,

whereas at temperatures $T<T_{c}$ our one-step approximation predicts $\omega=0$ due to the disappearance of the radicals. The value of $T_{c}$ is very dependent on the local $\mathrm{H}_{2}$ content through $\alpha$, 
which equals one for $Y_{\mathrm{H}_{2}}=0$ but decreases rapidly towards $\alpha=1 / 6$ when evaluated with the small nonzero values of $Y_{\mathrm{H}_{2}}$ typically found in the reaction zone. Because of the relatively large activation temperature of reaction $1 f, T_{a_{1 f}}=8590 \mathrm{~K}$, the overall rate $(9)$ is very sensitive to changes in temperature through the term $k_{1 f} /\left(\alpha k_{4 f} C_{\mathrm{M}}\right)$ appearing in the cutoff factor. The associated value of the dimensionless activation energy $\beta=T_{a_{1 f}} / T+n_{1 f}$ $-n_{4 f}+1$, written with account taken of the additional algebraic temperature dependences $T^{n_{1 f}}$ and $T^{n_{4 f}-1}$ of $k_{1 f}$ and $k_{4 f} C_{\mathrm{M}}$, where $n_{1 f}=-0.7$ and $n_{4 f}=-1.4$, is found to be of order 10 at temperatures of the order of the crossover temperature $T_{c}$. Therefore, changes in the reaction rate by a factor of order unity occur with small temperature increments of the order $\left(T-T_{c}\right) / T_{c} \sim \beta^{-1}$, which are enough to accommodate the changes in burning rate per unit surface found for increasing $\phi$ in the branch of stable flame balls, as explained below. It is of interest that, although the rate (9) was derived in connection with lean premixed deflagrations close to the flammability limit, it applies in general provided heat losses are strong enough to keep the flame temperature close to the crossover value.

In this one-step approximation, only three chemical species $\left(\mathrm{H}_{2}, \mathrm{O}_{2}\right.$ and $\left.\mathrm{H}_{2} \mathrm{O}\right)$ need to be considered. An additional simplification follows from noting that $\mathrm{d} Y_{\mathrm{H}_{2}}+\alpha_{\mathrm{H}_{2}} Y_{\mathrm{H}_{2}} \mathrm{~d} T / T=T^{-\alpha_{\mathrm{H}_{2}}} \mathrm{~d}$ $\left(T^{\alpha_{\mathrm{H}_{2}}} Y_{\mathrm{H}_{2}}\right)$ which allows the thermal diffusion and molecular diffusion terms to be incorporated in a single Fickian-like diffusion term written as a function of the modified fuel mass fraction

$Y=\left(T / T_{\infty}\right)^{\alpha_{\mathrm{H}_{2}}} Y_{\mathrm{H}_{2}}$

and with a modified diffusivity

$D=\left(T / T_{\infty}\right)^{-\alpha_{\mathrm{H}_{2}}} D_{\mathrm{H}_{2}}$.

The new transport operator, which is particularly useful for the analysis of convection-free steady configurations, can be used in (2) to write

$$
\begin{aligned}
\frac{1}{r^{2}} \frac{\mathrm{d}}{\mathrm{d} r}\left(\frac{\rho D_{\mathrm{O}_{2}}}{W_{\mathrm{O}_{2}}} r^{2} \frac{\mathrm{d} Y_{\mathrm{O}_{2}}}{\mathrm{~d} r}\right) & =-\frac{1}{r^{2}} \frac{\mathrm{d}}{\mathrm{d} r}\left(\frac{\rho D_{\mathrm{H}_{2} \mathrm{O}}}{2 W_{\mathrm{H}_{2} \mathrm{O}}} r^{2} \frac{\mathrm{d} Y_{\mathrm{H}_{2} \mathrm{O}}}{\mathrm{d} r}\right) \\
& =\frac{1}{r^{2}} \frac{\mathrm{d}}{\mathrm{d} r}\left(\frac{\rho D}{2 W_{\mathrm{H}_{2}}} r^{2} \frac{\mathrm{d} Y}{\mathrm{~d} r}\right) \\
& =\omega
\end{aligned}
$$

for the stoichiometry of the global reaction $2 \mathrm{H}_{2}+\mathrm{O}_{2} \rightarrow 2 \mathrm{H}_{2} \mathrm{O}$, whereas the energy equation reduces to

$\frac{1}{r^{2}} \frac{\mathrm{d}}{\mathrm{d} r}\left(\lambda r^{2} \frac{\mathrm{d} T}{\mathrm{~d} r}\right)=Q_{R}-2 W_{\mathrm{H}_{2}} q \omega$,

where $q=-h_{\mathrm{H}_{2} \mathrm{O}}^{o} / W_{\mathrm{H}_{2}}=1.21 \times 10^{5} \mathrm{~kJ} / \mathrm{kg}$ is the amount of heat released per unit mass of fuel consumed. Results obtained with this one-step description by integrating Eqs. (13) and (14) with boundary conditions $\mathrm{d} T / \mathrm{d} r=\mathrm{d} Y_{i} / \mathrm{d} r=0 \quad$ at $r=0 \quad$ and $\quad T-T_{\infty}=Y-Y_{\mathrm{H}_{2 \infty}}=Y_{\mathrm{O}_{2}}-Y_{\mathrm{O}_{2 \infty}}=$
$Y_{\mathrm{H}_{2} \mathrm{O}}=0$ as $r \rightarrow \infty$ are indicated by the dashed curves in Figs. 1 and 2, with the flame-ball radius in Fig. 1 defined as the location where the temperature reaches its crossover value. As can be seen, the resulting curves are practically indistinguishable from those obtained with the 21-step mechanism, indicating that the steady state is an excellent approximation for all chemical intermediates.

As previously mentioned, for $\phi>0.0735$ there exists a branch of unstable solutions, not depicted in Figs. 1 and 2, corresponding to flame balls that are smaller and hotter than those of the stable branch described herein. The flame balls along this unstable branch are sufficiently small for radiation to have a negligible effect. As the mixture becomes leaner, the decreasing reaction rate requires a larger flame-ball radius to ensure an adequate conduction-reaction balance in $\mathrm{Eq}$. (14). For conditions such that the peak temperature $T_{\max }$ approaches $T_{c}$ the reaction rate given by (9) becomes very small and the flame-ball radius of the conduction-reaction solution increases rapidly. With an increasing radius, the effect of radiant energy loss takes over and produces the transition to the branch of stable solutions through the turning point shown in Figs. 1 and 2. Because of the large value of the activation energy present in $\omega$, the peak temperature $T_{\max }$ of the resulting solutions cannot be far from the crossover value (i.e., if $T_{\max }-T_{c}$ were much larger than $\beta^{-1} T_{c}$, the associated increase in chemical reaction would be so large that it could not be balanced by radiation losses). Therefore, for increasing equivalence ratios, the resulting flame-ball radius for the branch of stable solutions increases in such a way that radiant energy losses can balance the chemical heat release while keeping the flame temperature at a value not far from $T_{c}$, a condition that determines at leading order the flame-ball structure, as seen below.

\section{The thin reaction-layer description}

For large nondimensional activation energies $\beta \gg 1$, the reaction occurs, as shown in Fig. 3, in a thin layer, where the fuel mass fraction and the temperature variations from the crossover value are of order $Y_{\mathrm{H}_{2}} / Y_{\mathrm{H}_{2 \infty}} \sim\left(T-T_{c}\right) / T_{c} \sim$ $\beta^{-1} \ll 1$; then, a reaction-sheet approximation can be used for describing the solution.

\subsection{Relationships between the chemical species}

For simplicity, we shall use the assumption, not strictly necessary, of equal temperature dependence of the diffusivities $D, D_{\mathrm{O}_{2}}$ and $D_{\mathrm{H}_{2} \mathrm{O}}$. Then, the first two equations in (13) can easily be integrated, with the boundary conditions (3) and (4), to yield the two simple relations 


$$
\begin{aligned}
Y_{\mathrm{H}_{2} \mathrm{O}} & =2 \frac{W_{\mathrm{H}_{2} \mathrm{O}}}{W_{\mathrm{O}_{2}}} \frac{D_{\mathrm{O}_{2}}}{D_{\mathrm{H}_{2} \mathrm{O}}}\left(Y_{\mathrm{O}_{2 \infty}}-Y_{\mathrm{O}_{2}}\right) \\
& =\frac{W_{\mathrm{H}_{2} \mathrm{O}}}{W_{\mathrm{H}_{2}}} \frac{D}{D_{\mathrm{H}_{2} \mathrm{O}}}\left(Y_{\mathrm{H}_{2 \infty}}-Y\right)
\end{aligned}
$$

giving the mass fractions of water vapor and oxygen in terms of the modified fuel mass fraction $Y$. In this approximation, the effect of thermal diffusion is incorporated by using in evaluating Eq. (15) an averaged increased diffusivity $D / D_{\mathrm{H}_{2}}=$ 1.154 , yielding

$\frac{Y_{\mathrm{H}_{2} \mathrm{O}_{r}}}{Y_{\mathrm{H}_{2 \infty}}}=\frac{W_{\mathrm{H}_{2} \mathrm{O}}}{W_{\mathrm{H}_{2}}} \frac{D}{D_{\mathrm{H}_{2} \mathrm{O}}}=28.73$

and

$$
\begin{aligned}
\frac{Y_{\mathrm{O}_{2 r}}}{Y_{\mathrm{O}_{2 \infty}}} & =1-\frac{1}{2} \frac{W_{\mathrm{O}_{2}}}{W_{\mathrm{H}_{2}}} \frac{D}{D_{\mathrm{O}_{2}}} \frac{Y_{\mathrm{H}_{2 \infty}}}{Y_{\mathrm{O}_{2 \infty}}} \\
& =1-\phi / 0.234,
\end{aligned}
$$

for the water vapor and oxygen mass fractions in the reaction zone, obtained from (15) with $Y=0$. In view of this last equation, it is clear that the lean regime of flame balls is restricted to configurations with $\phi<D_{\mathrm{O}_{2}} / D \simeq 0.234$, such that $Y_{\mathrm{O}_{2 r}}>0$, a result of the differential diffusion first pointed out by Joulin [15]. Note that this limiting equivalence ratio, corresponding to the effective stoichiometric conditions for which both reactants are simultaneously depleted in the reaction sheet, is clearly affected by thermal diffusion, which, according to the approximation $D / D_{\mathrm{H}_{2}}=1.154$ employed in evaluating the water vapor and oxygen at the flame, increases the transport rate of hydrogen by approximately $15 \%$, thereby also influencing the corresponding limiting value at the lean flammability limit. For larger equivalence ratios $\phi>0.234$, oxygen becomes the deficient reactant at the reaction layer. Then, because of the lower molecular diffusivity of $\mathrm{O}_{2}$, the flameball temperature drops rapidly from the value achieved when the much more diffusive species $\mathrm{H}_{2}$ is the limiting reactant, as shown in Fig. 2.

\subsection{The dimensionless formulation}

For given ambient conditions, the computation of $T_{\max }$ and $r_{f}$ requires integration of the last equation in (13) together with the energy equation (14) with the boundary conditions given above. The problem can be formulated in dimensionless form by scaling the variables according to $\theta=T / T_{\max }, y=Y / Y_{\mathbf{H}_{2 \infty}}$ and $\eta=r / r_{f}$ to give

$$
\begin{aligned}
& \frac{1}{\eta^{2}}\left(\theta^{v} \eta^{2} \theta^{\prime}\right)^{\prime}=\varepsilon(1-y) \frac{\theta^{4}-\theta_{\infty}^{4}}{\theta^{2}}-Q \Omega, \\
& \frac{1}{\eta^{2}}\left(\theta^{\gamma} \eta^{2} y^{\prime}\right)^{\prime}=\Omega,
\end{aligned}
$$

with the prime / denoting differentiation with respect to the coordinate $\eta$. The exponents for the temperature dependence of the transport coefficients are $v=0.85$ and $\gamma=0.99$ as dictated by Eqs. (5), (6) and (12), with $c_{p}$ assumed in Eq. (5) to be only a function of the temperature with a weak dependence $c_{p} \propto T^{0.15}$, as corresponds approximately to air. Here,

$$
\begin{aligned}
& \Omega=\frac{2 W_{\mathrm{H}_{2}} r_{f}^{2} \theta_{\infty}^{\gamma}}{(\rho D)_{\infty} Y_{\mathrm{H}_{2 \infty}}} \omega \text { and } \\
& Q=\frac{q Y_{\mathrm{H}_{2 \infty}} \theta_{\infty}^{1+v-\gamma}}{T_{\infty} \lambda_{\infty} /(\rho D)_{\infty}}
\end{aligned}
$$

are the dimensionless reaction rate and its corresponding heat release. In the dimensionless formulation, the original unknowns $T_{\max }$ and $r_{f}$ have been replaced with the ambient-to-flame temperature ratio

$\theta_{\infty}=T_{\infty} / T_{\max }$

and with the value of the ratio of the characteristic values of the local volumetric heat losses by radiation and conduction in the hot flame region

$\varepsilon=4 \kappa_{\mathrm{H}_{2} \mathrm{O}_{r}} \sigma p\left(W_{\text {air }} / W_{\mathrm{H}_{2} \mathrm{O}}\right) Y_{\mathrm{H}_{2} \mathrm{O}_{r}} T_{\max }^{3} r_{f}^{2} / \lambda_{r}$,

calculated with $Y_{\mathrm{H}_{2} \mathrm{O}_{r}}$, as given in Eq. (16), with the reaction-zone values $\kappa_{\mathrm{H}_{2} \mathrm{O}_{r}}$ and $\lambda_{r}$ evaluated at $T=T_{\max }$, and with the constant molecular weight of air $W_{\text {air }}$ replacing $W$, a reasonably accurate approximation for the lean conditions explored herein. Eqs. (18) and (19) are to be integrated with boundary conditions $\theta^{\prime}(0)=y^{\prime}$ $(0)=0$ and $\theta(\infty)-\theta_{\infty}=y(\infty)-1=0$. The additional conditions that the peak value of $\theta$ be $\theta=1$ and that $\theta=\left(T_{c} / T_{\infty}\right) \theta_{\infty}$ at $\eta=1$ are needed to determine the unknowns $\theta_{\infty}$ and $\varepsilon$.

\subsection{Solution with the reaction-sheet approximation}

For large values of the nondimensional activation energy $\beta$, the reaction-rate terms in (18) and (19) can be replaced by dirac-delta functions located at $\eta=1$ that cause the gradients of temperature and reactant to have a discontinuity at the flame according to

$\theta_{f-}^{\prime}-\theta_{f+}^{\prime}=Q y_{f+}^{\prime}$.

The inner-side gradient $y_{f-}^{\prime}$ can be neglected above since $y$ remains constant for $\eta<1$. In fact, it takes a value $y=0$, because the temperature gradient on the inner side of the flame sheet $\theta^{\prime}(1)=\theta_{f-}^{\prime}$ is not large enough to freeze the reaction, so that for $0<\eta<1$ the mixture is in near equilibrium with $y=0$. Integration of $\mathrm{Eq}$. (18) for $0<\eta<1$ with $\Omega=0$ and $y=0$, and with boundary conditions $\theta^{\prime}(0)=0$ and $\theta(1)=1$ gives the temperature distribution inside the flame ball, including the value of $\theta_{f-}^{\prime}$. Similarly, integration of Eqs. (18) and (19) with $\Omega=0$ for $\eta>1$ with boundary conditions $\theta(1)-1=y(1)=0$ and $\theta(\infty)-\theta_{\infty}=y(\infty)-1=0$ determines the distri- 
butions of $\theta(\eta)$ and $y(\eta)$ in the outer frozen region, including the gradients of temperature and fuel mass fraction outside the flame $\theta^{\prime}(1)=\theta_{f+}^{\prime}$ and $y^{\prime}(1)=y_{f+}^{\prime}$, whose variation with $\varepsilon$ as well as that of $\theta_{f-}^{\prime}$ are given in Fig. 4 for two representative values of $\theta_{\infty}$.

For $\beta \gg 1$, in the leading-order approximation corresponding to the limit $\beta \rightarrow \infty$, the reaction layer appears as infinitesimally thin, and the peak temperature and the crossover temperature take the same leading-order value $T_{c}=T_{\max }=T_{c}^{0}$, where $T_{c}^{0}$ is the crossover temperature obtained for a given equivalence ratio $\phi$ by solving $(10)$ with use made of the reaction-zone composition given in (16) and (17) and with $\alpha=1$, as corresponds to taking $Y_{\mathrm{H}_{2}}=0$ in the reaction zone, giving the results shown in Fig. 2 as a thin dot-dashed curve. The corresponding value of $\theta_{\infty}^{0}=T_{\infty} / T_{c}^{0}$ can be used in computing the gradients $\theta_{f-}^{\prime}, \theta_{f+}^{\prime}$, and $y_{f+}^{\prime}$ as functions of $\varepsilon$, to be substituted into (23) to provide an implicit relation that can be solved for $\varepsilon$. These leading-order results can be used in (22) to determine the corresponding flame-ball radius $r_{f}$, which is plotted in Fig. 1.

Relative corrections to $T_{\max }$, of order $\beta^{-1}$, can be obtained by analyzing the quasi-planar reaction layer; the analysis will also provide the value of the fuel mass fraction $Y_{c} \sim \beta^{-1} Y_{\mathrm{H}_{2 \infty}}$ at the crossover point where reaction (9) freezes, to be employed in evaluating the function $\alpha<1$ in (10), which in turn determines the corrected crossover temperature $T_{c}$. The amount of fuel burnt per unit time can be determined by integrating (19) across the reaction layer with the reaction rate $\Omega$ evaluated with the equilibrium mass frac-

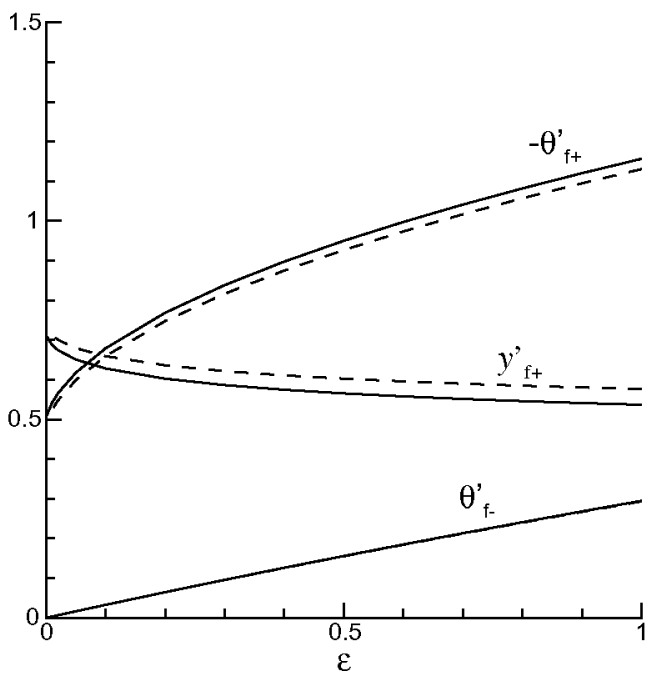

Fig. 4. The variation with $\varepsilon$ of $y_{f+}^{\prime}, \theta_{f-}^{\prime}$, and $\theta_{f+}^{\prime}$ for $\theta_{\infty}=0.25$ (solid curves) and $\theta_{\infty}=0.30$ (dashed curves) as obtained from integrations of Eqs. (18) and (19) as described in the text. tions of water vapor and oxygen given in Eqs. (16) and (17). In the integration, the temperature must be computed in terms of $Y$ as dictated by

$1-\theta=Q y-\theta_{f-}^{\prime}(\eta-1)$,

obtained by integrating twice the chemistry-free equation obtained by adding (18) to the product of (19) times $Q$. The solution is greatly simplified when the effect of heat losses is neglected by discarding the last term in (24), an approximation that is justified by the relatively small values of $\theta_{f-}^{\prime}$ seen in Fig. 4. Under those conditions, integration of (19) after multiplication by $\mathrm{d} y / \mathrm{d} \eta$ yields $\left(y_{f+}^{\prime}\right)^{2}=2 \int_{0}^{y_{c}} \Omega \mathrm{d} y$, which can be written as

$\left(y_{f+}^{\prime}\right)^{2} /\left(\frac{4 W_{\mathbf{H}_{2}} r_{f}^{2} \theta_{\infty}^{y}}{(\rho D)_{\infty} Y_{\mathrm{H}_{2 \infty}}}\right)=\int_{0}^{Y_{c}} \omega \mathrm{d} Y$,

where $y_{f+}^{\prime}, r_{f}$, and $\theta_{\infty}$ can be evaluated in terms of the leading-order results, and the integral on the right-hand side can be performed with use made of (24) expressed in the simplified form $1-T / T_{\max }=Q Y / Y_{\mathrm{H}_{2 \infty}}$. Solving simultaneously the three Eqs. (10), (25) and $1-T_{c} / T_{\max }=$ $Q Y_{c} / Y_{\mathrm{H}_{2 \infty}}$ determines the corrected values of $T_{\max }$ and $T_{c}$ as well as the fuel mass fraction at the crossover point $Y_{c}$. The resulting value of $T_{\max }$ is shown in Fig. 2 as a thick dot-dashed curve.

As can be seen in Figs. 1 and 2, the analytical solution describes with remarkable accuracy the whole branch of stable solutions. Errors in the flame-ball radii are typically smaller than $10 \%$. On the other hand, the leading-order prediction for the peak temperature $T_{\max }=T_{c}^{0}$ contains errors on the order of $100 \mathrm{~K}$, but these errors reduce considerably when the first-order correction is introduced by accounting for the solution within the reaction layer, yielding overpredictions of peak temperatures that are always below $30 \mathrm{~K}$. Note that the approximations used for the analytical results necessarily limit their accuracy, both for small flame balls approaching extinction, where the reaction region can be anticipated to extend all the way to the center of the flame ball, thereby invalidating the flame-sheet approximation, and also for very large flame balls with significant radiation; these are such that the temperature gradient appearing on the inner side of the flame sheet may quench the chemical reaction there, producing significant fuel leakage, a higher-order effect not accounted for in the present analysis, which neglects the last term in Eq. (24). The associated departures are however small, so that the reaction-sheet description is satisfactory, as demonstrated by the plots in Figs. 1 and 2 .

\section{Conclusions}

While steady, freely propagating planar flames in hydrogen-air mixtures at normal atmospheric 
conditions exist only for equivalence ratios above a lean flammability limit $\phi_{l}=0.251[13,14]$, determined by the condition that the flame temperature equals the crossover temperature $T_{c}$, nonpropagating flames in the form of flame balls exist under microgravity conditions for much lower values of the equivalence ratio due to the high diffusivity of hydrogen enhanced by Soret effects. These flame balls are stable due to radiation, with a radius that increases in such a way that radiant heat losses keep the peak temperature at a value that barely exceeds $T_{c}$. Under those conditions, the one-step chemistry recently developed for near-limit hydrogen-air deflagrations $[13,14]$ can be used to describe with excellent accuracy the whole branch of stable flame balls. As may be seen in Fig. 1, while different theoretical results agree well with each other, experimental results for flame-ball radii exhibit a considerable amount of scatter, possibly due to unsteady effects in the flame-initiation stage. Further well-controlled microgravity flame-ball experiments therefore would appear to be worthwhile.

\section{Acknowledgements}

This work was supported by the Spanish MCINN through Project \# ENE2008-06515 and by the Comunidad de Madrid through Project \# S2009/ENE-1597.

[1] P. Saxena, F.A. Williams, Combust. Flame 145 (2006) 316-323.
[2] F.A. Williams, J.F. Grcar, Proc. Combust. Inst. 32 (2009) 1351-1357.

[3] P.D. Ronney, Proc. Combust. Inst. 27 (1998) 2485 2506.

[4] J. Buckmaster, M. Smooke, V. Giovangigli, Combust. Flame 94 (1993) 113-124.

[5] M-S. Wu, P.D. Ronney, R.O. Colantonio, D.M. VanZandt, Combust. Flame 116 (1999) 387-397.

[6] Available at: <http://maemail.ucsd.edu/combustion/cermech>.

[7] M.D. Smooke, V. Giovangigli, in: M.D. Smooke (Ed.), Reduced Kinetic Mechanisms and Asymptotic Approximations for Methane-Air Flames, Lecture Notes in Physics, vol. 384, Springer-Verlag, Berlin, 1991, pp. 1-28.

[8] R.M. Fristrom, L. Monchick, Combust. Flame 71 (1988) 89-99.

[9] W.G. Vincenti, C.H. Kruger, Introduction to Physical Gas Dynamics, Wiley, New York, 1967, pp. 491-495.

[10] R.S. Barlow, A.N. Karpetis, J.H. Frank, J.-Y Chen, Combust. Flame 127 (2001) 2102-2118.

[11] P.D. Ronney, K.N. Whaling, A. Abbud-Madrid, J.L. Gatto, V.L. Pisowicz, AIAA J. 32 (1994) 569-577.

[12] M-S Wu, J-B. Liu, P.D. Ronney, Proc. Combust. Inst. 27 (1998) 2543-2550.

[13] D. Fernández-Galisteo, A.L. Sánchez, A. Liñán, F.A. Williams, Combust. Flame 156 (2009) 985996.

[14] D. Fernández-Galisteo, A.L. Sánchez, A. Liñán, F.A. Williams, Combust. Theoret. Model. 13 (4) (2009) 741-761.

[15] G. Joulin, SIAM J. Appl. Math. 47 (1987) 998 1016. 